\title{
Auroral Contribution to Sky Brightness for Optical Astronomy on the Antarctic Plateau
}

\author{
J. T. Dempsey ${ }^{\mathrm{A}}$, J. W. V. Storey ${ }^{\mathrm{A}, \mathrm{B}}$, and A. Phillips ${ }^{\mathrm{A}}$ \\ A School of Physics, University of New South Wales, Sydney, NSW 2052, Australia \\ ${ }^{B}$ Corresponding author. Email: j.storey@unsw.edu.au
}

Received 2004 April 8, accepted 2004 December 15

\begin{abstract}
The Antarctic Plateau holds great promise for optical astronomy. One relatively unstudied feature of the polar night sky for optical astronomical observing is the potential contamination of observations by aurorae. In this study we analyse auroral measurements at South Pole Station and show that during an average winter season, the auroral contribution to the $B$ band sky brightness is below $21.9 \mathrm{~B} \mathrm{mag} \mathrm{arcsec}-2$ for $50 \%$ of the observing time. In $V$ band, the median sky brightness contribution is $20.8 \mathrm{mag} \mathrm{arcsec}^{-2}$ during an average winter. South Pole Station is situated within the auroral zone and experiences strong and frequent auroral activity. The Antarctic locations of Dome C and Dome A are closer to the geomagnetic pole where auroral activity is greatly reduced compared with that of South Pole Station. Calculations based on satellite measurements of electron flux above the Antarctic Plateau are used to show that at Dome $\mathrm{C}$, the contribution to sky background in the $B$ and $V$ bands is up to 3.1 mag less than that at the South Pole. The use of notch filters to reduce the contribution from the strongest auroral emission lines and bands is also discussed. The scientific potential of an extremely large telescope located at Dome $\mathrm{C}$ is discussed, with reference to the effect that auroral emissions would have on particular astronomical observations.
\end{abstract}

Keywords: atmospheric effects — site testing

\section{Introduction}

Auroral emission over Antarctica has been studied intensively for the last 50 years by physicists. During the last 20 years, satellite data have provided a nearly continuous stream of images of the auroral activity within the auroral zone, an oval-shaped region which is generally centred on the geomagnetic south pole. Ions and electrons ejected from the sun's corona are transported towards the Earth as the solar wind. Aurorae are a result of collisions between precipitating charged particles (mostly electrons) and atmospheric gases. Each gas (oxygen and nitrogen molecules and atoms) emits characteristic spectral lines when bombarded. Atmospheric composition varies with altitude with predominantly molecular gas at low altitude giving way to atomic gas at higher altitude.

Since the faster precipitating particles penetrate deeper into the atmosphere and affect different atomic and molecular species, the colour of the aurora is partly dependent on the incoming particle energy spectrum. The auroral altitude range extends between 80 and $1000 \mathrm{~km}$, with typical aurorae occurring at 100 to $250 \mathrm{~km}$ above the ground. The colour of the typical aurora is yellow-green, from a transition of atomic oxygen, which emits at a wavelength of $557.7 \mathrm{~nm}$. Auroral light from lower levels in the atmosphere is dominated by blue and red bands from molecular nitrogen and molecular oxygen. Above $250 \mathrm{~km}$, auroral light is characterised by a red spectral line of atomic oxygen at $630 \mathrm{~nm}$.

Bright auroral emissions are mostly confined to an elliptically-shaped region centred on the geomagnetic pole, known as the auroral oval. This zone ranges from about $-75^{\circ}$ geomagnetic latitude at local noon, to about $-67^{\circ}$ geomagnetic latitude at midnight under average conditions. An image of the southern auroral oval, with the positions of the South Pole, Dome C, and Dome A indicated, is presented in Figure 1.

The geomagnetic pole location is determined by calculation using magnetic reference field models (MacMillan \& Quinn 2000). This location provides a constant reference point for geophysical study of the aurora throughout a given year of observation. At the beginning of 2004, the position of the geomagnetic south pole was $79.3^{\circ} \mathrm{S}$, $108.5^{\circ} \mathrm{E}$, a shift of $0.24^{\circ}$ southward and $0.1^{\circ}$ eastward from 2003.

The aim of this paper is to evaluate the contribution of auroral emissions to the overall photometric and spectroscopic sky brightness above the polar plateau, and the consequences for optical astronomy. The three sites on the Antarctic Plateau that will be considered are South Pole Station, Dome C, and Dome A. South Pole is located at a geomagnetic latitude of $-76^{\circ} 13^{\prime}$, which is directly under the dayside auroral oval. Dome A is closer to the geomagnetic pole at a geomagnetic latitude of $-81^{\circ} 58^{\prime}$, 


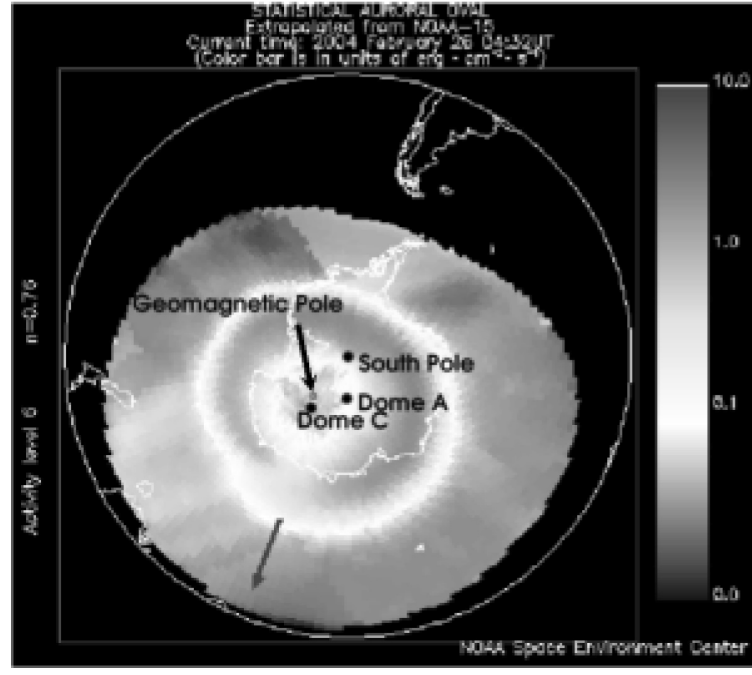

Figure 1 South Pole, Dome C, and Dome A locations placed on an image of the southern auroral oval, from measurements taken during a recent polar orbit of an NOAA POES satellite, 2004 February 26. Activity index $K \mathrm{p}=6$ (high) (NOAA Space Weather Updates, www. sec.noaa.gov/pmap).

Table 1. Potential optical astronomy sites on the high Antarctic plateau, and their geomagnetic (gmt) coordinates, calculated for 2004

\begin{tabular}{llcrr}
\hline Potential Site & Latitude & Longitude & $\begin{array}{c}\text { gmt } \\
\text { Latitude }\end{array}$ & $\begin{array}{c}\text { gmt } \\
\text { Longitude }\end{array}$ \\
\hline South Pole & $-90^{\circ}$ & $-00^{\circ}$ & $-76^{\circ} 13^{\prime}$ & $11^{\circ} 53^{\prime}$ \\
Dome C & $-75^{\circ} 06^{\prime}$ & $123^{\circ} 23^{\prime}$ & $-89^{\circ} 10^{\prime}$ & $264^{\circ} 56^{\prime}$ \\
Dome A & $-81^{\circ}$ & $81^{\circ}$ & $-81^{\circ} 58^{\prime}$ & $51^{\circ} 10^{\prime}$ \\
Skibotn & $69^{\circ} 22^{\prime}$ & $20^{\circ} 19^{\prime}$ & $66^{\circ} 27^{\prime}$ & $105^{\circ} 24^{\prime}$ \\
\hline
\end{tabular}

${ }^{1}$ Skibotn is a Norwegian observatory situated outside the northern auroral zone. and Dome $\mathrm{C}$ is situated very near the geomagnetic south pole itself at a geomagnetic latitude of $-89^{\circ} 10^{\prime}$. The geographic and 2004 geomagnetic coordinates for these sites are presented in Table 1.

\subsection{Auroral Line Intensities and Spatial Extent}

One measure of auroral intensity is the International Brightness Coefficient (IBC) which is scaled to the intensity of the 557.7-nm forbidden oxygen [OI] emission line, on a logarithmic scale from IBC1 $\left(I_{557.7 \mathrm{~nm}}=1 \mathrm{kR}\right)$ to IBC4 $\left(I_{557.7 \mathrm{~nm}}=1000 \mathrm{kR}\right)$ (Vallance Jones 1974). Most of the calculations in the following sections follow model calculations from Vallance Jones (1974), assuming a bright aurora of intensity IBC3, corresponding to an intensity of $100 \mathrm{kR}$ for the 557.7-nm [OI] line. An IBC3 aurora is exceptionally bright and occurs very rarely, but such luminosity is often tabulated as a reference brightness.

A high resolution spectrum of some of the visible wavelength auroral emission lines is presented in Figure 2 (Swenson et al. 1998). Between wavelengths of 400 and $600 \mathrm{~nm}$, the $427.8-\mathrm{nm} \mathrm{N}_{2}^{+}$band emission and the 557.7-nm [OI] line make the most significant contributions. At $630.0 \mathrm{~nm}$ and $636.4 \mathrm{~nm}$ are located [OI] transitions, and at longer wavelengths the spectrum is dominated by molecular oxygen and $\mathrm{N}_{2}$ band emission. An example showing the time evolution of intensities of the 427.8-nm (blue), 557.7-nm (green), and 630.0-nm (red) lines is presented in Figure 3.

The intensities are given in rayleighs (R), a common unit used by auroral physicists when describing auroral brightness (Chamberlain 1961). By definition, $1 \mathrm{R}=7.96 \times 10^{8}$ photons $\mathrm{s}^{-1} \mathrm{~m}^{-2} \mathrm{sr}^{-1}$ (Baker \& Romick 1976).

If we consider a $1 \mathrm{kR}$ emission at $557.7 \mathrm{~nm}$, we can calculate the intensity when observed through a $V$ band filter (i.e., centred at $556 \mathrm{~nm}$ and with a bandwidth of $85 \mathrm{~nm}$ ). If

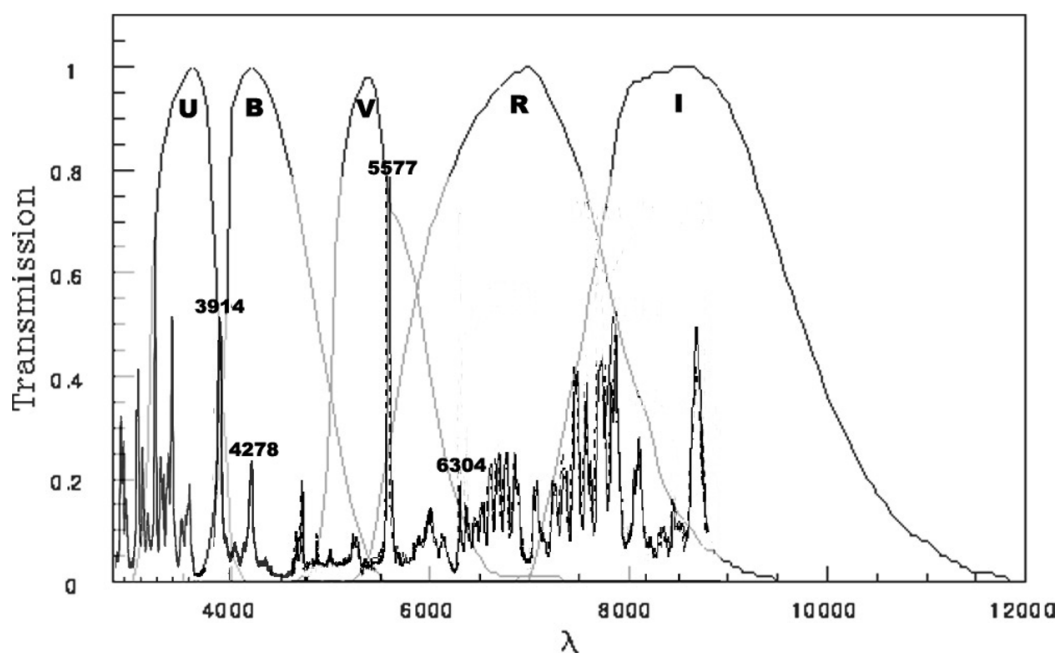

Figure 2 The position of the visible auroral lines with respect to the UBVRI passbands. This particular auroral spectrum was acquired during a bright auroral event, and covers wavelengths from 300 to $890 \mathrm{~nm}$ (Swenson et al. 1998; Paxton \& Meng 1999). 


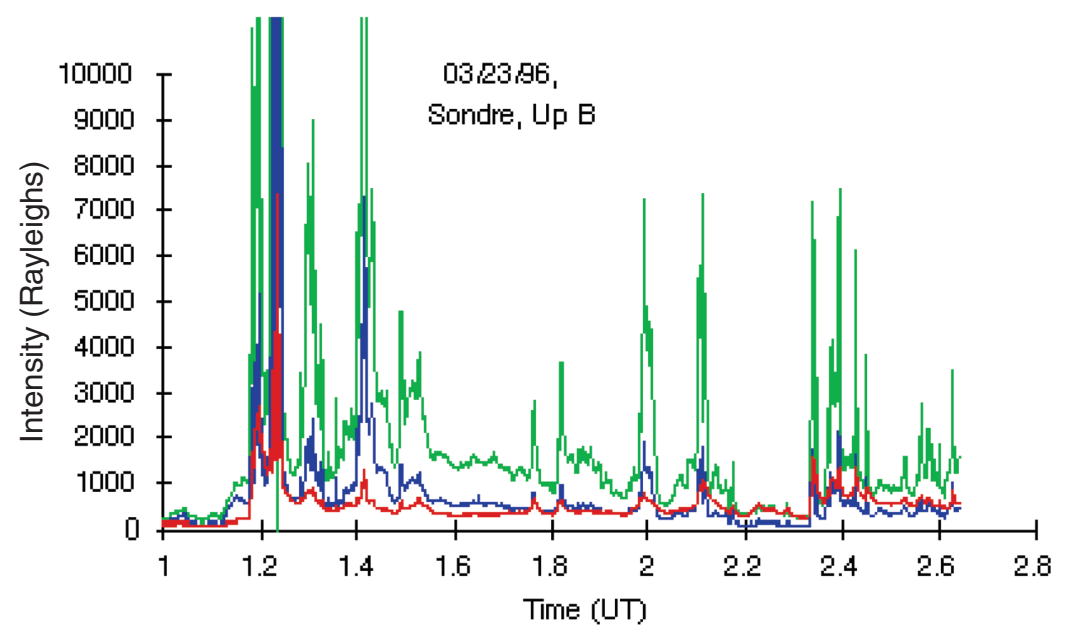

Figure 3 Time history intensity for the 427.8-nm (blue), 557.7-nm (green), and 630.0-nm (red) auroral emission event shown in Figure 2 (Swenson et al. 1998).

the total flux from the 557.7-nm line were distributed as a continuum uniformly across the $V$ band, it would, at each wavelength, be equal to $11.8 \mathrm{R} \mathrm{nm}^{-1}$. This intensity, $I$, in $\mathrm{R} \mathrm{nm}^{-1}$, may then be converted to a flux in $\mu$ janskys per square arcsecond at the central wavelength of the band, using the equation (Benn \& Ellison 1998)

$$
F=I \times\left(1.24 \times 10^{6}\right) \times \lambda,
$$

where $\lambda$ is the central wavelength in metres, and the resulting flux, $F$, is in $\mu$ janskys per square arcsecond. The flux may then be converted to $V$ magnitudes per square arcsecond by the equation

$$
\text { magnitudes } \operatorname{arcsec}^{-2}=20-2.5 \log _{10}\left(\frac{F}{K}\right),
$$

where $F$ is the auroral flux in the band measured in microjanskys per square arcsecond, and $K$ is equal to $42.6,36.4$, and 30.8 for the $B, V$, and $R$ bands respectively (Bessel 1979; Benn \& Ellison 1998). Therefore a $1 \mathrm{kR}$ auroral emission at $557.7 \mathrm{~nm}$ when observed in $V$ band has a brightness of $21.6 \mathrm{mag} \operatorname{arcsec}^{-2}$. Likewise for a $B$ band filter (centred at $436 \mathrm{~nm}$ with a bandwidth of $94 \mathrm{~nm}$ ), a $1 \mathrm{kR}$ emission at $427.8 \mathrm{~nm}$ is equivalent to $5.75 \mu \mathrm{Jy} \operatorname{arcsec}^{-2}$, or a $B$ brightness of $22.2 \mathrm{mag} \mathrm{arcsec}^{-2}$.

For the particular auroral spectrum presented in Figure 2, intensities of $7000 \mathrm{R}$ at $557.7 \mathrm{~nm}, 2000 \mathrm{R}$ at $427.8 \mathrm{~nm}$, and $800 \mathrm{R}$ at $630.0 \mathrm{~nm}$ were recorded. A list of auroral line and molecular band emission intensities, relative to a $557.7-\mathrm{nm}$ emission line of strength $100 \mathrm{kR}$, is presented in Tables 2-8 (Omholt 1971; Vallance Jones 1974) for an IBC3 aurora (these intensities will be used for calculations in Section 5). Vallance Jones (1974) states that the ratio of the 427.8-nm band intensity to the 557.7-nm line intensity is always close to a figure of 0.33 , whilst the ratio of the 557.7-nm to the 630.4-nm line intensities can range between 0.02 and 1 . The $427.8-\mathrm{nm} \mathrm{N}_{2}^{+}$band emission and 557.7-nm [OI] line emission are dependent only on the incident electron flux, whereas the relative intensity of the 630.4 and 636.4-nm [OI] lines is also determined by the energy spectrum of the incident electrons (Vallance Jones 1974).

For an IBC3 aurora producing the intensities shown in Tables 2-8, calculations show a sky brightness contribution of $16.6 \mathrm{~V} \mathrm{mag} \operatorname{arcsec}^{-2}$ from the 557.7-nm line, $18.5 \mathrm{~B} \mathrm{mag} \operatorname{arcsec}^{-2}$ for the $427.8-\mathrm{nm} \mathrm{N}_{2}^{+}$band emission, and $17.0 \mathrm{R}$ mag $\operatorname{~arcsec}^{-2}$ from the $630.0-\mathrm{nm}$ [OI] line (assuming the maximum emission of $100 \mathrm{kR}$ ). The $630.0-\mathrm{nm}$ emission line is somewhat weaker on average than the 557.7-nm [OI] line and the 427.8-nm band emission intensities (Vallance Jones 1974).

\subsection{Previous Studies}

There are no large optical astronomical observatories located at high northern latitudes, where auroral light is a dominant contributor to sky brightness at visible wavelengths. The only observatory where auroral light has a significant impact on observations is a small observatory close to Skibotn, Norway. Skibotn is situated within the most active region of the auroral oval (see Figure 1). Studies during 1979-1984 by Myrabo $(1979,1980)$ investigated the viability of stellar photometry in this region of strong and variable auroral activity.

Myrabo used a double-aperture chopping photometer (operating at $2-20 \mathrm{~Hz}$ ), with fields of view of $3^{\prime}$ each, and care was taken to ensure the throughputs of both fields were identical. An auroral photometer, with a $1^{\circ}$ field of view and a 2.0-nm bandpass centred on $427.8 \mathrm{~nm}$, observed the same stellar field as the telescope, permitting subtraction of the measured auroral brightness during the observation. Over three years of observations, the mean sky brightness measured in the $U B V$ colour bands was found to be $U=17.2, B=18.8$, and $V=18.1$, all in star magnitudes per square arcsecond (Myrabo 1980).

Myrabo's papers also show that the 427.8-nm band emission is a very good indicator of the total sky brightness in the $B$ band, although it is subject to rapid variation in its brightness. Rapid changes in auroral intensity and 
Table 2. Emission line intensities for the observed forbidden atomic multiplets

\begin{tabular}{lll}
\hline Emitter & $\lambda[\mathrm{nm}]$ & Intensity $[\mathrm{kR}]$ \\
\hline$[\mathrm{OI}]$ & 297.2 & 6 \\
{$[\mathrm{OI}]$} & 557.7 & 100 \\
{$[\mathrm{OI}]$} & $630.0-636.4$ & $2-100$ \\
{$[\mathrm{OII}]$} & $373.7-372.9$ & 1 \\
{$[\mathrm{OII}]$} & $731.9-733.0$ & $0.4-100$ \\
{$[\mathrm{NI}]$} & 346.6 & 1 \\
{$[\mathrm{NI}]$} & $519.9-520.1$ & $0.1-2$ \\
{$[\mathrm{NI}]$} & $1039.5-1040.4$ & 6 \\
\hline
\end{tabular}

Table 3. Emission line intensities for the observed allowed atomic multiplets

\begin{tabular}{llllcc}
\hline Emitter & $\lambda[\mathrm{nm}]$ & $\begin{array}{c}\text { Intensity } \\
{[\mathrm{kR}]}\end{array}$ & Emitter & $\lambda[\mathrm{nm}]$ & $\begin{array}{c}\text { Intensity } \\
{[\mathrm{kR}]}\end{array}$ \\
\hline OI & 374.9 & 1 & NI & 493.5 & 0.19 \\
OI & 397.3 & 0.3 & NI & 532.9 & 0.22 \\
OI & 436.8 & 0.5 & NI & 644.2 & 0.11 \\
OI & 441.5 & 0.5 & NI & 746.8 & 0.55 \\
OI & 459.1 & 0.13 & NI & 82.6 & 3.3 \\
OI & 464.9 & 0.6 & NI & 862.9 & 3.84 \\
OI & 595.9 & 0.13 & NI & 868.0 & 10.5 \\
OI & 615.8 & 0.14 & NII & 399.5 & 1 \\
OI & 645.4 & 0.14 & NII & 404.4 & 0.5 \\
OI & 700.2 & 0.3 & NII & 424.4 & 0.5 \\
OI & 777.4 & 9.6 & NII & 500.1 & 0.6 \\
OI & 799.5 & 1.2 & NII & 568.0 & 0.65 \\
OI & 844.6 & 11.5 & NII & 648.2 & 0.4 \\
\hline
\end{tabular}

Table 4. Emission band intensities for the ${ }^{1} \mathrm{~N} \mathrm{~N}_{2}^{+}$band

\begin{tabular}{cccccc}
\hline$\lambda[\mathrm{nm}]$ & $\begin{array}{c}\text { Intensity } \\
{[\mathrm{kR}]}\end{array}$ & $\lambda[\mathrm{nm}]$ & $\begin{array}{c}\text { Intensity } \\
{[\mathrm{kR}]}\end{array}$ & $\lambda[\mathrm{nm}]$ & $\begin{array}{c}\text { Intensity } \\
{[\mathrm{kR}]}\end{array}$ \\
\hline 330.8 & 0.02 & 419.9 & 0.05 & 470.9 & 6.04 \\
356.3 & 0.15 & 423.6 & 3.87 & 514.9 & 0.32 \\
358.2 & 6.80 & 427.8 & 30.0 & 522.8 & 0.99 \\
388.4 & 3.93 & 459.9 & 0.03 & 575.4 & 0.06 \\
391.4 & 98.51 & 465.2 & 1.34 & 586.5 & 0.14 \\
\hline
\end{tabular}

Table 5. Emission band intensities for the $\mathrm{M} \mathrm{N}_{2}^{+}$band

\begin{tabular}{lccccc}
\hline$\lambda[\mathrm{nm}]$ & $\begin{array}{c}\text { Intensity } \\
{[\mathrm{kR}]}\end{array}$ & $\lambda[\mathrm{nm}]$ & $\begin{array}{c}\text { Intensity } \\
{[\mathrm{kR}]}\end{array}$ & $\lambda[\mathrm{nm}]$ & $\begin{array}{c}\text { Intensity } \\
{[\mathrm{kR}]}\end{array}$ \\
\hline 504.8 & 0.05 & 785.3 & 65.49 & 1277 & 2.28 \\
517.4 & 0.08 & 792.4 & 0.11 & 1326 & 0.8 \\
530.5 & 0.08 & 808.1 & 38.91 & 1379 & 0.20 \\
544.2 & 0.06 & 823.3 & 10.99 & 1460 & 36.67 \\
552.9 & 0.33 & 857.8 & 2.50 & 1521 & 32.00 \\
567.1 & 0.46 & 884.5 & 0.85 & 1581 & 10.06 \\
581.9 & 0.37 & 912.7 & 0.02 & 1654 & 1.40 \\
597.4 & 0.22 & 918.1 & 158.0 & 1728 & 0.05 \\
612.3 & 2.11 & 947.0 & 64.43 & 2126 & 3.21 \\
628.5 & 2.43 & 977.4 & 9.63 & 2240 & 5.77 \\
645.5 & 1.55 & 1010 & 0.36 & 2363 & 3.84 \\
663.3 & 0.74 & 1044 & 0.05 & 2498 & 1.40 \\
681.9 & 0.29 & 1080 & 0.15 & 2648 & 1.40 \\
687.3 & 14.22 & 1110 & 122.69 & 2813 & 0.08 \\
701.6 & 0.10 & 1118 & 0.11 & 3856 & 0.08 \\
706.4 & 10.50 & 1147 & 10.06 & 4186 & 0.19 \\
726.3 & 5.23 & 1158 & 0.05 & 4578 & 0.17 \\
747.2 & 1.84 & 1188 & 1.84 & 5016 & 0.08 \\
769.3 & 0.51 & 1231 & 4.67 & 5552 & 0.03 \\
\hline
\end{tabular}

Table 6. Emission band intensities for the ${ }^{1} \mathbf{P} \mathbf{N}_{2}$ band

\begin{tabular}{cccccc}
\hline$\lambda[\mathrm{nm}]$ & $\begin{array}{c}\text { Intensity } \\
{[\mathrm{kR}]}\end{array}$ & $\lambda[\mathrm{nm}]$ & $\begin{array}{c}\text { Intensity } \\
{[\mathrm{kR}]}\end{array}$ & $\lambda[\mathrm{nm}]$ & $\begin{array}{c}\text { Intensity } \\
{[\mathrm{kR}]}\end{array}$ \\
\hline 575.5 & 0.21 & 738.7 & 23.74 & 1051 & 96.36 \\
580.4 & 0.43 & 750.4 & 48.70 & 1078 & 4.57 \\
585.5 & 0.75 & 761.2 & 0.96 & 1117 & 1.03 \\
590.6 & 1.28 & 762.7 & 63.56 & 1152 & 3.24 \\
595.9 & 1.80 & 775.2 & 1.94 & 1159 & 0.97 \\
601.4 & 2.36 & 775.4 & 53.61 & 1193 & 26.41 \\
606.9 & 2.27 & 789.7 & 3.17 & 1237 & 54.88 \\
612.7 & 1.76 & 804.8 & 3.01 & 1306 & 12.68 \\
618.7 & 0.75 & 820.6 & 1.04 & 1363 & 12.68 \\
625.3 & 0.29 & 837.0 & 0.90 & 1427 & 21.13 \\
632.3 & 0.81 & 854.2 & 17.84 & 1489 & 0.89 \\
639.5 & 2.14 & 872.3 & 74.50 & 1498 & 17.27 \\
646.8 & 4.52 & 891.2 & 124.5 & 1571 & 5.20 \\
654.5 & 8.93 & 898.3 & 1.35 & 1664 & 8.80 \\
662.4 & 13.5 & 920.3 & 6.613 & 1706 & 0.97 \\
670.5 & 18.2 & 943.6 & 17.130 & 1769 & 8.12 \\
678.9 & 17.5 & 968.0 & 23.439 & 1887 & 3.81 \\
687.5 & 8.80 & 984.2 & 1.58 & 1962 & 3.03 \\
706.0 & 0.27 & 993.9 & 15.09 & 2121 & 3.27 \\
716.5 & 2.24 & 1013 & 3.36 & 2306 & 2.00 \\
727.4 & 8.36 & 1045 & 5.26 & & \\
\hline & & & & & \\
\hline
\end{tabular}

Table 7. Emission band intensities for the $\mathrm{O}_{2}$ atmospheric band

\begin{tabular}{lccrcc}
\hline$\lambda[\mathrm{nm}]$ & $\begin{array}{c}\text { Intensity } \\
{[\mathrm{kR}]}\end{array}$ & $\lambda[\mathrm{nm}]$ & $\begin{array}{c}\text { Intensity } \\
{[\mathrm{kR}]}\end{array}$ & $\lambda[\mathrm{nm}]$ & $\begin{array}{c}\text { Intensity } \\
{[\mathrm{kR}]}\end{array}$ \\
\hline 596.0 & 0.02 & $761.9^{1}$ & 1176.1 & 906.2 & 1.63 \\
628.9 & 0.05 & 770.8 & 13.96 & 918.0 & 1.93 \\
637.1 & 0.38 & 780.2 & 6.79 & 996.8 & 1.44 \\
645.7 & 0.29 & 790.1 & 11.53 & 1007 & 0.07 \\
654.8 & 0.56 & 800.3 & 3.04 & 1018 & 0.09 \\
688.2 & 1.66 & 811.1 & 2.25 & 1030 & 0.33 \\
697.0 & 1.93 & 864.5 & 58.00 & 1042 & 0.19 \\
706.0 & 6.09 & 874.2 & 1.61 & 1055 & 0.30 \\
715.4 & 2.75 & 884.4 & 1.40 & 1173 & 0.02 \\
725.3 & 3.44 & 895.1 & 3.89 & 1235 & 0.02 \\
\hline
\end{tabular}

${ }^{1}$ The $761.9-\mathrm{nm}(0,0)$ transition is not observable from the ground because of self-absorption by ground state $\mathrm{O}_{2}$ (Vallance Jones 1974).

Table 8. Emission band intensities for the ${ }^{1} \mathrm{NO}_{2}^{+}$band

\begin{tabular}{llllcc}
\hline$\lambda[\mathrm{nm}]$ & $\begin{array}{c}\text { Intensity } \\
{[\mathrm{kR}]}\end{array}$ & $\lambda[\mathrm{nm}]$ & $\begin{array}{c}\text { Intensity } \\
{[\mathrm{kR}]}\end{array}$ & $\lambda[\mathrm{nm}]$ & $\begin{array}{c}\text { Intensity } \\
{[\mathrm{kR}]}\end{array}$ \\
\hline 498.7 & 0.2 & 632.1 & 0.38 & 785.4 & 0.33 \\
525.2 & 0.90 & 638.9 & 3.47 & 799.1 & 0.04 \\
527.4 & 1.884 & 665.7 & 0.08 & 813.7 & 0.12 \\
554.2 & 0.06 & 673.5 & 0.86 & 829.8 & 0.21 \\
557.3 & 1.08 & 682.2 & 1.90 & 847.5 & 0.12 \\
560.8 & 5.90 & 700.5 & 0.02 & 857.2 & 0.02 \\
585.7 & 0.23 & 709.7 & 0.19 & 875.6 & 0.07 \\
590.0 & 0.65 & 719.7 & 0.74 & 896.0 & 0.09 \\
594.7 & 0.23 & 730.7 & 0.85 & 918.3 & 0.04 \\
599.9 & 3.98 & 747.1 & 0.04 & 945.9 & 0.03 \\
620.4 & 0.07 & 758.7 & 0.18 & 971.6 & 0.04 \\
625.9 & 0.02 & 771.4 & 0.43 & 1000 & 0.02 \\
\hline & & & & &
\end{tabular}


spatial extent cause high-frequency variations in observed brightness. Fast-moving aurorae and rapid brightening can cause variations from a few kilorayleighs up to several hundreds of kilorayleighs within a fraction of a minute (Myrabo 1980). High-speed photometric measurements (McHarg 1998) observe the rapid variation in flickering aurorae, and although most observed intensity fluctuations are below a frequency of $80 \mathrm{~Hz}$, frequencies as high as $180 \mathrm{~Hz}$ are detected. Deep-field long-duration exposures, and sky background subtraction, will obviously be compromised by such rapidly varying features.

Auroral arcs can also possess fine spatial structure (Trondsen 1998). Arcs are narrow linear auroral features that can range from about $100 \mathrm{~m}$ across down to structures as small as $10 \mathrm{~m}$ within the ionosphere. Bright, dynamic arcs of this scale can also be observed embedded in more diffuse airglow (Trondsen 1998). Small scale structures appear even in narrow field-of-view observations and cannot be removed by simple background subtraction.

Auroral structures do not often dominate the entire sky, but even when observing unilluminated regions, a bright aurora outside of a telescope's field of view will contribute to the sky brightness (Gattinger et al. 1991). The high ground albedo of the Antarctic Plateau (Carroll \& Fitch 1981) increases this effect.

\section{AFOS Measurements}

The Antarctic Fibre Optic Spectrometer (AFOS), which is part of the Automated Astronomical Site Testing Observatory (Storey, Ashley, \& Burton 1996), has collected data at South Pole Station for periods during the winters of 2000,2002 , and a large part of 2003. The AFOS is a $30-\mathrm{cm}$ Newtonian telescope that passes its collected light through a dichroic beamsplitter and into two fibre optic bundles, each bundle comprising three optical fibres. One bundle is optimised for red light transmission, and the other for blue-UV transmission. The fibres are $40 \mathrm{~m}$ in length and terminate in a spectrometer. The ends of the fibres act as a pseudo-slit for a 200 lines $\mathrm{mm}^{-1}$ grating spectrometer, which then projects the light onto a $1024 \times 256$-pixel CCD array. The fibres have a diameter of $100 \mu \mathrm{m}$ and each collects light from a 19.6" diameter spot on the sky. The wavelength range of the spectrometer is $300-850 \mathrm{~nm}$, with a spectral resolution of $2.4 \mathrm{~nm}$. Further details of the instrument can be found in Boccas et al. (1998).

The AFOS instrument acquired stellar measurements throughout the winters of 2002 and 2003, mostly with $<60$ s exposures of very bright and well-known stars (magnitude $<2.0$ ). The primary goal of the instrument was to investigate the atmospheric extinction at the South Pole. None of these measurements had sufficient sensitivity to detect auroral lines, although comparison with auroral data from South Pole shows that there were occasions when there was auroral activity in the sky. Only one set of data, taken with AFOS pointed at the moon during the full lunar eclipse of 2000 July 16, shows auroral lines evident in the spectra. During the peak of the eclipse a strong auroral storm occurred directly over the face of the moon, affecting spectra collected by the AFOS for a period of approximately $40 \mathrm{~min}$ throughout eclipse totality. Only within the longest exposures taken during the full eclipse (of 100 and $600 \mathrm{~s}$ respectively) were the auroral lines detectable. An example of the AFOS spectrum is presented in Figure 4. The relatively low intensity of the blue emission lines is a result of lower transmission of the fibres towards UV wavelengths. This spectrum was acquired during complete eclipse and the solar spectrum (reflected off the moon) is only apparent in the far red wavelengths.

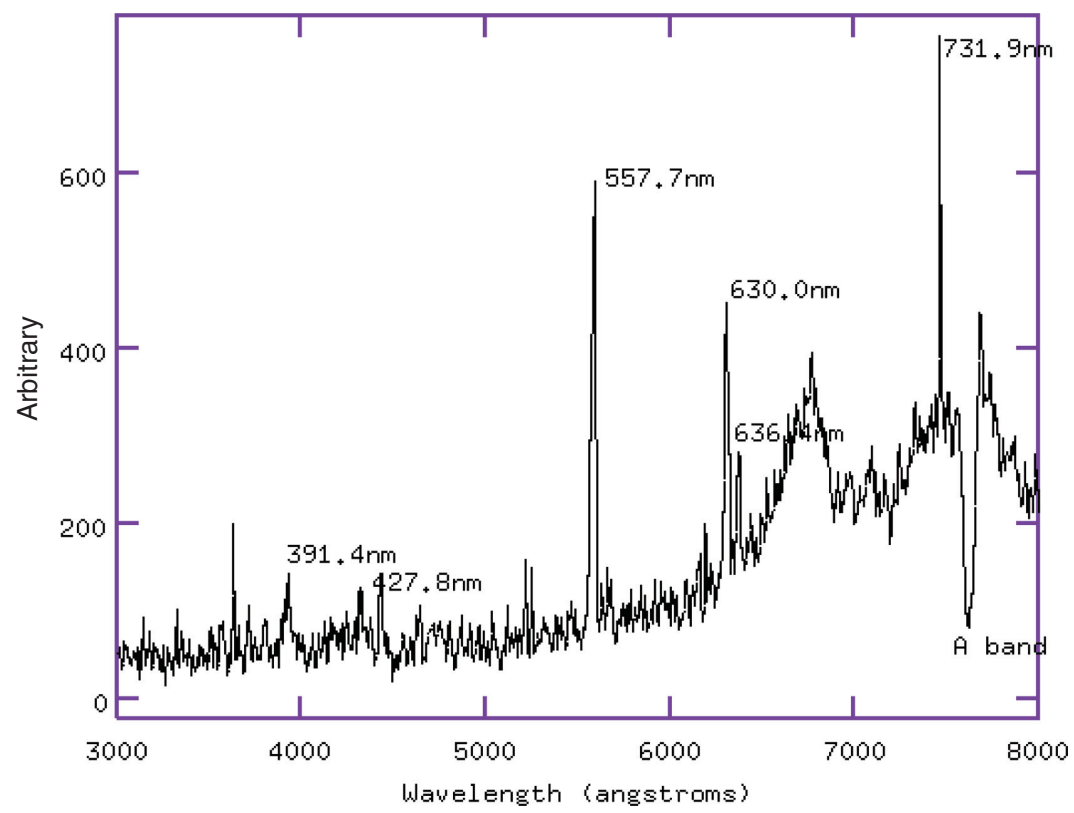

Figure 4 Spectrum of the moon observed during the total lunar eclipse of 2000 July 16 with the AFOS. Strong auroral lines can be seen. 


\section{Auroral Data from South Pole Station}

Figures 5 and 6 present auroral intensity data collected at South Pole Station (D. L. Detrick 2004, private communication). These data were acquired by two photometers, each having a field of view of approximately $60^{\circ}$, and used in conjunction with narrowband filters of bandwidth $3.0 \mathrm{~nm}$. One photometer measured the auroral emission at $427.8 \mathrm{~nm}$ (of the $\mathrm{N}_{2}^{+}$band), and the other measured at $630 \mathrm{~nm}$ ([OI] transition). The data used to generate Figures 5 and 6 were of $10 \mathrm{~min}$ mean intensities acquired during the entire winter periods (approximately 125 days) of 1985 (solar minimum) and 1990 (solar maximum). The 427.8-nm photometer had a limiting sensitivity of $23.7 \mathrm{~B}$ mag $\operatorname{arcsec}^{-2}$. The $630.0-\mathrm{nm}$ photometer limiting sensitivity is $24.5 R$ mag $\operatorname{arcsec}^{-2}$.

The intensity ratio of $I_{427.8} / I_{557.7}$ is generally fairly close to a figure of 0.33 (Vallance Jones 1974). Using this ratio, we have scaled the $I_{427.8}$ intensities to estimate the 557.7-nm emission. In our plots presenting the 427.8 and 557.7-nm intensity distributions, the units have been converted from rayleighs to visual magnitudes per square arcsecond, using the method outlined in Section 1.1. The histograms show the number of hours per winter that a particular intensity was observed.

From the percentile distribution curves shown in Figures 5 and 6 , we can conclude that for $50 \%$ of winter observing time during a solar minimum year, the emission intensity of the 557.7-nm line is less than $2000 \mathrm{R}$. For a $V$ band filter (centred at $556 \mathrm{~nm}$ and with bandwidth of $85 \mathrm{~nm}$ ), a $2 \mathrm{kR}$ emission at $557.7 \mathrm{~nm}$ is equivalent to $23.6 \mathrm{R} \mathrm{nm}^{-1}$ at each wavelength when averaged across the $V$ band. This implies a sky background brightness in $V$ of $20.9 V$ mag $\operatorname{arcsec}^{-2}$. During a solar maximum year, the median brightness is slightly lower at $21.7 \mathrm{Vmag} \operatorname{arcsec}^{-2}$. During a solar minimum year (if we consider a $B$ band centred at $436 \mathrm{~nm}$ and with a bandwidth of $94 \mathrm{~nm})$, the $427.8-\mathrm{nm} \mathrm{N}_{2}^{+}(0,1)$ band emission has an intensity of $22.8 \mathrm{~B} \mathrm{mag} \mathrm{arcsec}-2$, or less, for $50 \%$ of the winter period. During a solar maximum this becomes $23.5 \mathrm{~B} \mathrm{mag} \operatorname{arcsec}^{-2}$, or less, for $50 \%$ of observing time.

The 427.8 and 557.7-nm auroral line intensities are dependent on the electron flux incident on the atmosphere above the site (Vallance Jones 1974). The South Pole is positioned close to the inner edge of the quiet auroral oval. During solar maximum the auroral oval generally widens, extending to higher and lower geomagnetic latitudes. At such times the South Pole experiences lower electron flux and thus less intense aurorae whilst lower (geomagnetic) latitude sites experience increased auroral activity.

A histogram of the red 630.0-nm [OI] line photometer data is presented in Figure 7, and from this plot we conclude that for $50 \%$ of winter observing time during a solar minimum year, the emission intensity that the 630.0-nm line would produce when viewed through an $R$ filter is less than $24.6 R$ mag $\operatorname{arcsec}^{-2}$. During a solar maximum year, this median value becomes $25.4 R$ mag $\operatorname{arcsec}^{-2}$. Unlike the 427.8 and 557.7-nm emission, the 630.0-nm emission intensity is particularly dependent on the energy spectrum of the incident electrons (Vallance Jones 1974). There is relatively more 630.0-nm emission during solar maximum, indicating softer precipitation.

\section{Sky Brightness in Standard Photometric Bands}

In Figure 2, high-resolution spectra of auroral emission lines from Swenson et al. (1998) and Paxton \& Meng (1999) are superimposed upon the transmission curves for the UBVRI filter passbands. We will use spectra to estimate the contributions of auroral emission to sky background flux in the $B, V$, and $R$ bands, firstly when standard filters are used, and secondly when additional 'notch' filters are used to remove the strongest auroral lines and bands. We will consider the effect on sky observations when using a commercial holographic notch filter such as one manufactured by Kaiser Optical Systems (www.kosi.com/filters/notch.html). Another technique for producing extremely sharp, deep notch filters is the Rugate Method (Johnson \& Crane 1993).

Individual auroral lines are very narrow, with line widths dependent on the temperature of the atmosphere where the excitations occur. The Doppler-broadened FWHM of a single auroral line is of the order $<0.1 \mathrm{~nm}$ (Omholt 1971; Vallance Jones 1974). Molecular band emission, which is caused by vibrational excitation, is generally much broader, up to $\approx 5 \mathrm{~nm}$ (Bashkin et al. 1991). The data presented in Section 3 were acquired by photometers installed with filters of bandwidth $3 \mathrm{~nm}$.

The auroral line and band emission lists shown in Tables 2 to 8 indicate that there are a significant number of contributors to the auroral emission spectrum within the optical wavebands. In addition to this, observations show that between the predicted auroral emission bands and lines, a quasi-continuum emission is also seen (Gattinger \& Vallance Jones 1974; Sharp 1978). This emission is likely caused by weaker molecular bands of nitrogen and oxygen that lie below the resolution of the spectrometer (Bashkin 1991). For an IBC3 aurora, Gattinger \& Vallance Jones (1974) calculate a quasicontinuum level of $270 \mathrm{R} \mathrm{nm}^{-1}$ between 450 and $650 \mathrm{~nm}$, dropping to $100 \mathrm{R} \mathrm{nm}^{-1}$ at $900 \mathrm{~nm}$. In the following calculations (for an IBC3 aurora), we have included a quasi-continuum of $270 \mathrm{R} \mathrm{nm}^{-1}$.

\subsection{B Band}

In the $B$ band, the $\mathrm{N}_{2}^{+}(0,1)$ vibrational band emission (including $427.8 \mathrm{~nm}$ ) is the significant auroral contributor. All of our following calculations use the intensities shown in Tables 2 to 8 . Fortunately the stronger $\mathrm{N}_{2}^{+}(0,0)$ band emission centred at $391.4 \mathrm{~nm}$ lies beyond the edge of a typical $B$ band filter. The $\mathrm{N}_{2}^{+}(1,3)$ vibrational band at $465.2 \mathrm{~nm}, \mathrm{~N}_{2}^{+}(0,1)$ at $423.6 \mathrm{~nm}$, and $\mathrm{N}_{2}^{+}(0,2)$ at $470.9 \mathrm{~nm}$ are the strong emissions within the $B$ band.

For an IBC3 aurora, the quasi-continuum intensity within the $B$ band is $270 \mathrm{R} \mathrm{nm}^{-1}$, equivalent to $85 \%$ of 


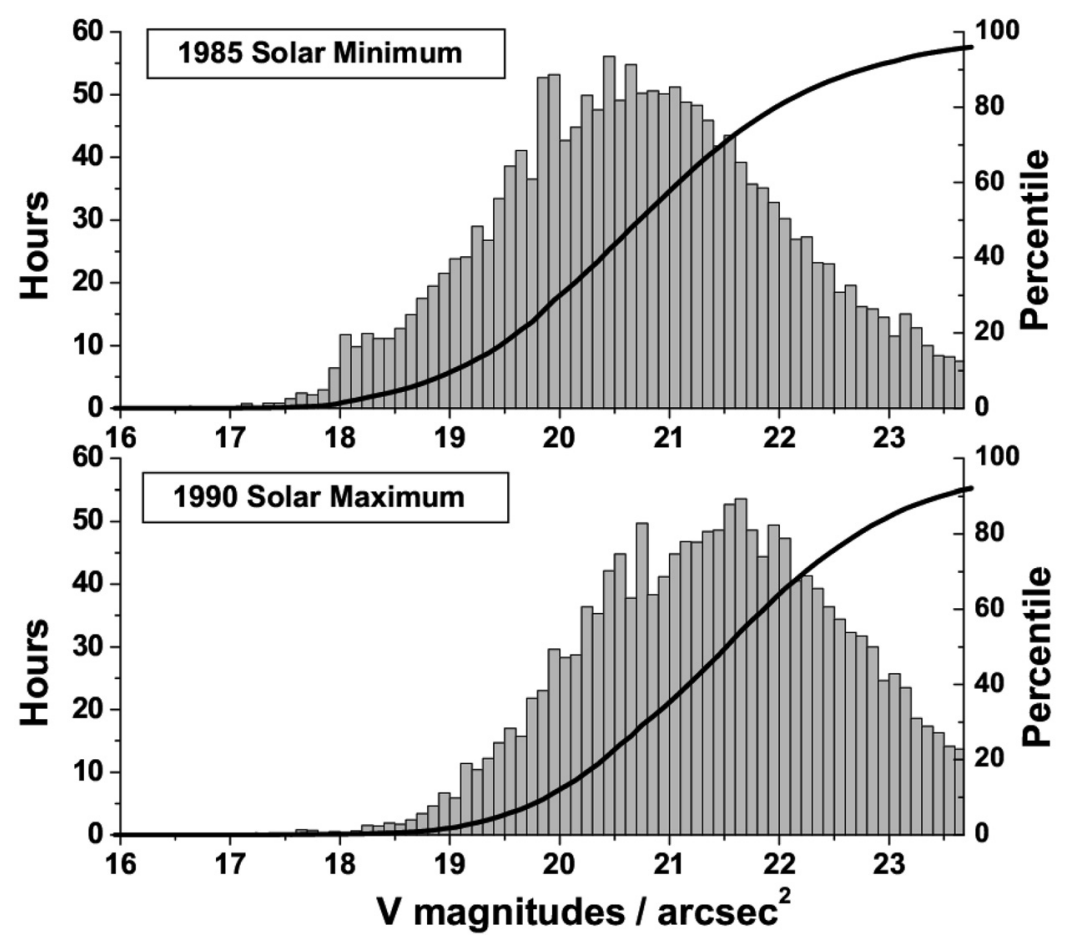

Figure 5 Intensity of the 557.7-nm [OI] line observed at the South Pole, in $V$ magnitudes per square arcsecond. The left axis shows the number of hours per winter that a particular intensity is observed. The cumulative probability is shown on the right-hand axis.

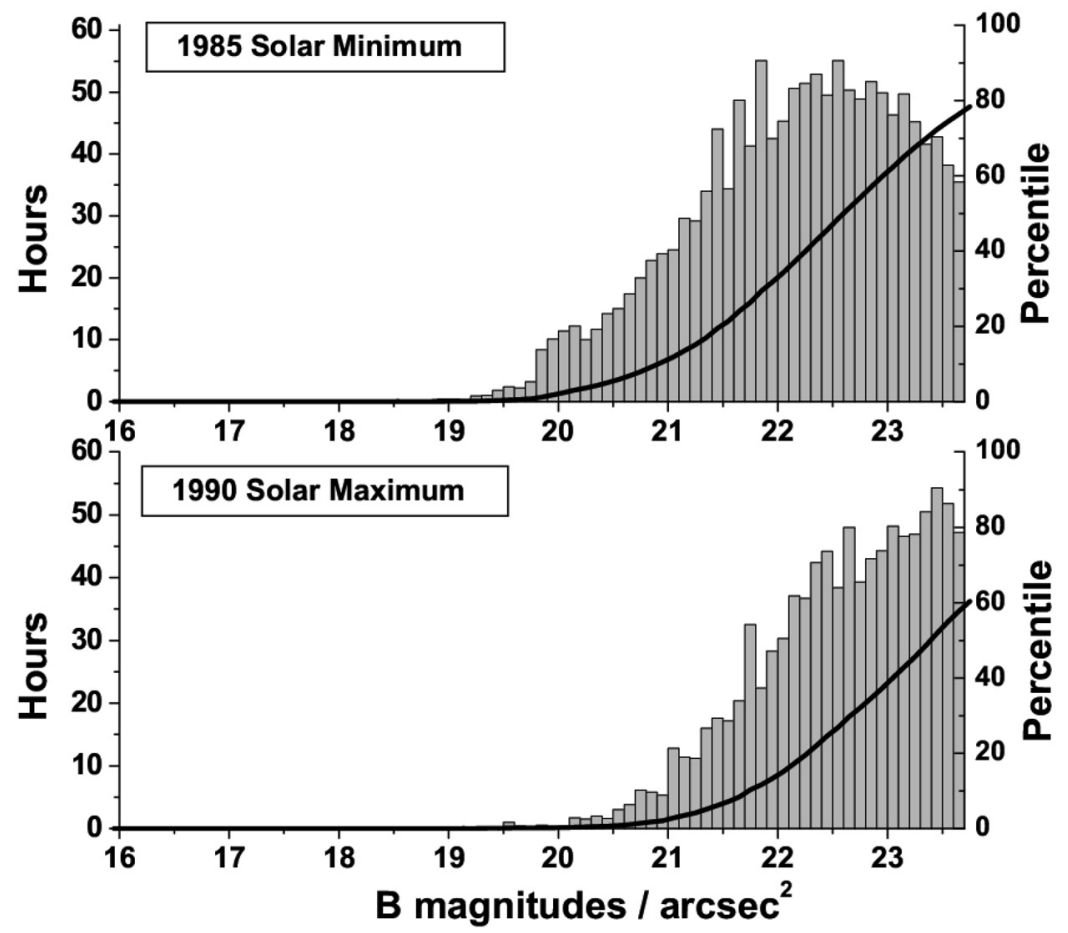

Figure 6 Intensity of the 427.8-nm nitrogen band observed at the South Pole, in $B$ magnitudes per square arcsecond. The left axis shows the number of hours per winter that a particular intensity is observed. The cumulative probability is shown on the right-hand axis.

the emission intensity at $427.8 \mathrm{~nm}$ (Gattinger \& Vallance Jones 1974). The intensity ratio, $I_{\text {quasi }} / I_{427.8}=0.85$, is therefore used to estimate the quasi-continuum contribution within the $B$ band from the 427.8 -nm photometer data.
A typical $B$ band filter has a central wavelength of $436.0 \mathrm{~nm}$ and an effective width of $94.0 \mathrm{~nm}$. Tables 2 to 8 show the intensities of the dominant auroral emission lines for an IBC3 aurora. For such an aurora, the 427.8-nm band 


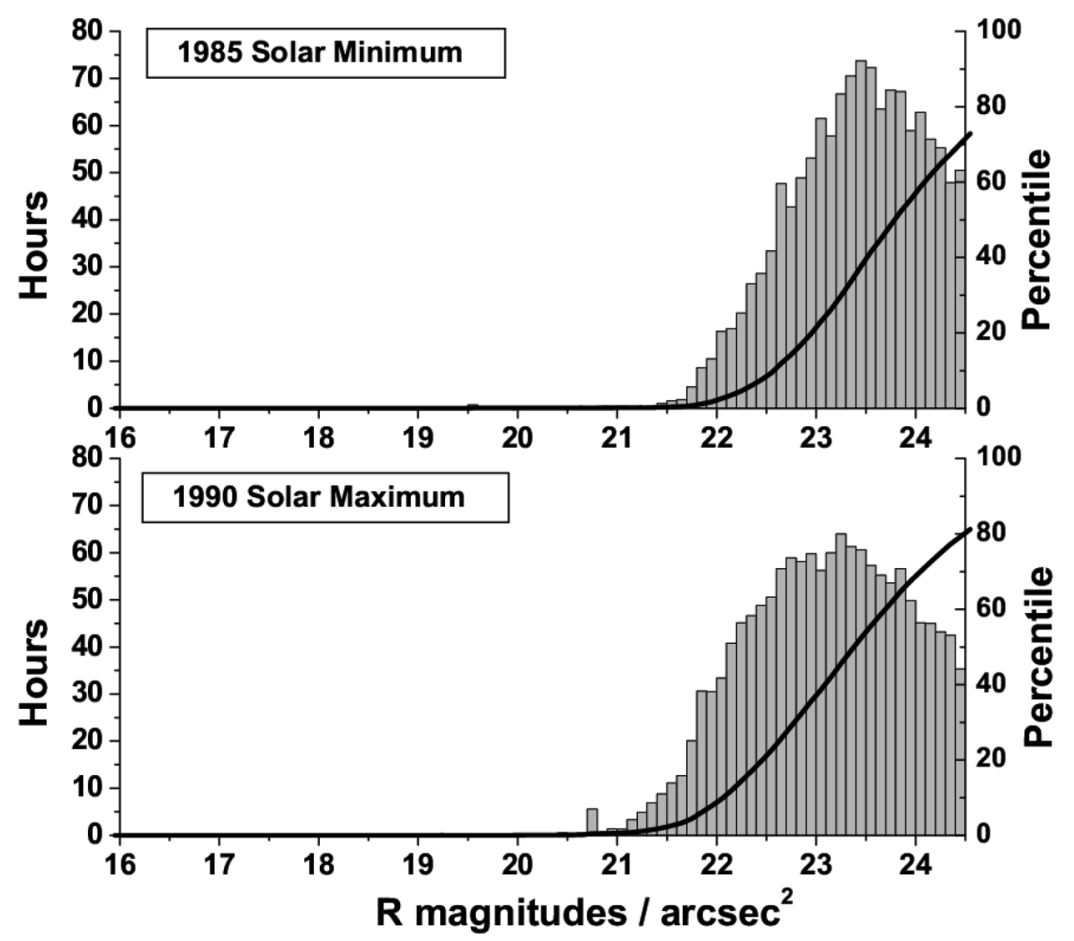

Figure 7 Intensity of the 630.0-nm [OI] line observed at the South Pole, in $R$ magnitudes per square arcsecond. The left axis shows the number of hours per winter that a particular intensity is observed. The cumulative probability is shown on the right-hand axis.

emission intensity is $30 \mathrm{kR}$ and the intensities of the other strong emission in this band produce a combined intensity of $11.3 \mathrm{kR}$. The total flux from these auroral emissions, if distributed as a continuum uniformly across the $B$ band, would, at each wavelength, be equal to $429 \mathrm{R} \mathrm{nm}^{-1}$. If we add to this a quasi-continuum contribution of $270 \mathrm{R} \mathrm{nm}^{-1}$, the emission from the auroral lines, bands, and continuum for an IBC3 aurora would be equivalent to $709 \mathrm{R} \mathrm{nm}^{-1}$ when averaged across the $B$ band. This results in a $B$ band auroral intensity of $17.6 \mathrm{~B} \mathrm{mag} \operatorname{arcsec}^{-2}$.

By scaling the 427.8-nm South Pole photometer measurements to estimate the total $B$ band auroral intensity we have calculated the probability distribution of $B$ magnitudes per square arcsecond for the winters of 1985 and 1990, years which correspond to the solar minimum and maximum respectively. This is represented by the solid grey histogram in Figure 8.

We now consider the use of a notch filter that has the effect of reducing the 427.8-nm flux measurements to negligible levels. The remaining emission bands and quasicontinuum have a combined intensity of $390 \mathrm{R} \mathrm{nm}^{-1}$ when averaged across the $B$ band. This results in a $B$ band intensity of $18.3 \mathrm{mag} \mathrm{arcsec}^{-2}$. When considering what depth of notch is required to reduce the contribution from the 427.8-nm line to below $10 \%$ of the $B$ band total, we find that an attenuation of 5 is sufficient.

Thus, a single notch filter centred at $427.8 \mathrm{~nm}$ could decrease the $B$ band background sky emission by $0.7 \mathrm{mag}$. This is shown as the hatched line histogram in Figure 8 .

\section{$4.2 \mathrm{~V}$ Band}

A standard $V$ band filter extends from wavelengths of 500 to $650 \mathrm{~nm}$ and includes the strongest auroral emission feature, the forbidden oxygen [OI] transition at $557.7 \mathrm{~nm}$. It is this particular emission that gives aurorae their characteristic green colour. Other lines included in the $V$ band are the $\mathrm{N}_{2}^{+}$vibrational bands at $522.8,612.3$, and $628.5 \mathrm{~nm}$, with relative intensities as shown in Tables 2 to 8.

For an IBC3 aurora, the quasi-continuum intensity in the $V$ band is $270 \mathrm{R} \mathrm{nm}^{-1}$, equivalent to $23 \%$ of the emission intensity at $557.7 \mathrm{~nm}$ (Gattinger \& Vallance Jones $1974)$. The intensity ratio $I_{\text {quasi }} / I_{557.7}=0.23$ is therefore used to estimate the quasi-continuum contribution in the $V$ band from the 557.7-nm photometer data.

A typical $V$ band filter is centred at $556.0 \mathrm{~nm}$, and has an effective width of $85.0 \mathrm{~nm}$. For an IBC3 aurora, the intensity of the 557.7-nm line is, by definition, $100 \mathrm{kR}$. The combined emission from the other strong emission bands within the $V$ band produce an intensity of $5.5 \mathrm{kR}$. The total flux from these auroral emissions, if distributed as a continuum uniformly across the band, would be equal to $1242 \mathrm{R} \mathrm{nm}^{-1}$. If we add to this a quasi-continuum contribution of $270 \mathrm{R} \mathrm{nm}^{-1}$, the emission from the auroral lines, bands, and continuum for an IBC3 aurora is equivalent to $1512 \mathrm{R} \mathrm{nm}^{-1}$ when averaged across the $V$ band. This results in a sky background intensity of $16.4 \mathrm{~V}$ mag $\operatorname{arcsec}^{-2}$.

If we consider using a notch filter with a FWHM of $10.0 \mathrm{~nm}$ that reduces the observed emission from the 557.7-nm [OI] line to negligible levels, the remaining 


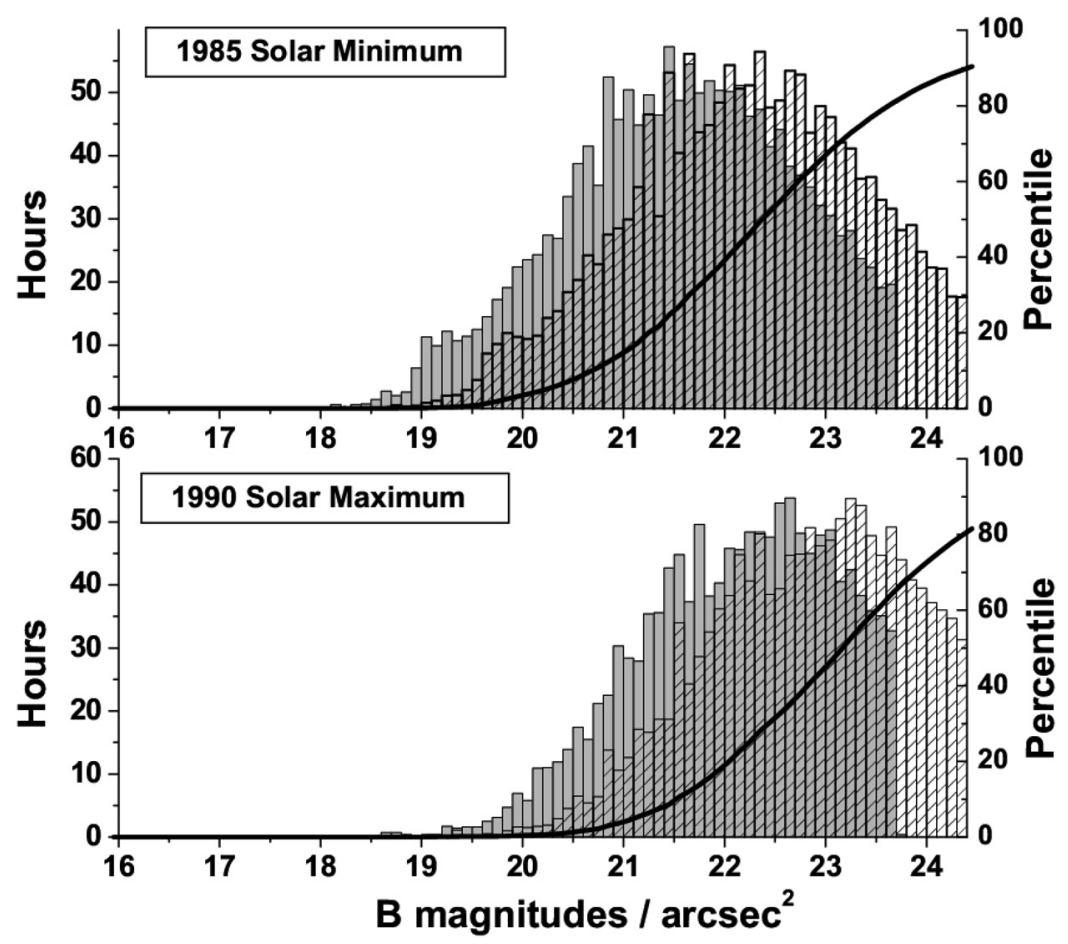

Figure 8 Sky brightness observed at the South Pole (in $B$ magnitudes per square arcsecond) for a standard $B$ filter (solid grey line) and for a notch filter at 427.8-nm (hatched line). The cumulative probability for the filter histogram is shown on the right-hand axis.

bands and quasi-continuum contribute emission equivalent to an intensity of $338 \mathrm{R} \mathrm{nm}^{-1}$. This is equivalent to a $V$ band brightness of $18.0 \mathrm{mag} \operatorname{arcsec}^{-2}$. In this case the notch filter would decrease the background by $1.6 \mathrm{mag}$. A filter with a notch attenuation of only 10 would suffice to reduce the 557.7-nm line to below $10 \%$ of the in-band total.

By using the tables of relative emission line strength (Tables 2 to 8), the South Pole 427.8-nm photometer measurements have been rescaled to estimate a probability distribution of the total $V$ band emission for the winters of 1985 and 1990. This is shown by the solid grey histogram in Figure 9. The calculations were then repeated for the case where a 557.7-nm notch filter is used, and this probability distribution is shown by the hatched line histogram in Figure 9.

\subsection{R Band}

It is clear from Figure 2 that within the $R$ band the removal of bright auroral lines is not as straightforward as for the $B$ or $V$ bands. The forbidden transitions of oxygen produce the 630.0 and 636.4-nm auroral lines which are less easy to categorise because their intensities vary quite widely, depending on the energy of the incident electrons and ions striking the upper atmosphere. It is certainly the case that these emissions are less frequent than the 557.7-nm emission (Omholt 1971; Vallance Jones 1974), and of a lower intensity than the emission lines in the blue and visible wavebands. However, in addition to the above lines, the $R$ band contains a number of other strong emission lines of $\mathrm{N}_{2}$, as well as lines of atomic and molecular oxygen (Omholt 1971). In addition, observations in the $R$ band are always affected by numerous atmospheric lines, since many of them are produced in airglow processes present above all ground-based astronomical sites.

The best ways to reduce $R$ band auroral emission depend on the type of observation being made. For example, consider the Vulcan South Transit Search project, currently operating at South Pole Station. Vulcan South Transit Search (Caldwell et al. 2002) is a wide-field imaging telescope observing $6 \mathrm{deg}^{-2}$ fields of sky, and searching for extrasolar planets. The waveband they are using is centred at $675.0 \mathrm{~nm}$, with a FWHM of $60 \mathrm{~nm}$. This waveband is a good compromise between avoiding the 630.0-nm red oxygen auroral emission line and the spectral distribution of night sky lines which begin at around $720 \mathrm{~nm}$. From 640 to $720 \mathrm{~nm}$ the primary band contributions are from first order $\mathrm{N}_{2}$ excitations, with a small contribution from atmospheric molecular oxygen (which increases towards the 760-nm oxygen atmospheric band).

Unlike the case of the $B$ and $V$ bands discussed previously, there is little to be gained by removing a few of these lines when such a narrow passband is already in use. Sky background within this narrow band is almost completely dominated by auroral emission.

\section{Auroral Intensity at Dome $\mathrm{C}$ and Dome $\mathrm{A}$}

Unlike the South Pole, there are as yet no ground-based measurements of auroral intensity at Dome $\mathrm{C}$ and Dome $\mathrm{A}$. However, there are satellite measurements (Hardy et al. 


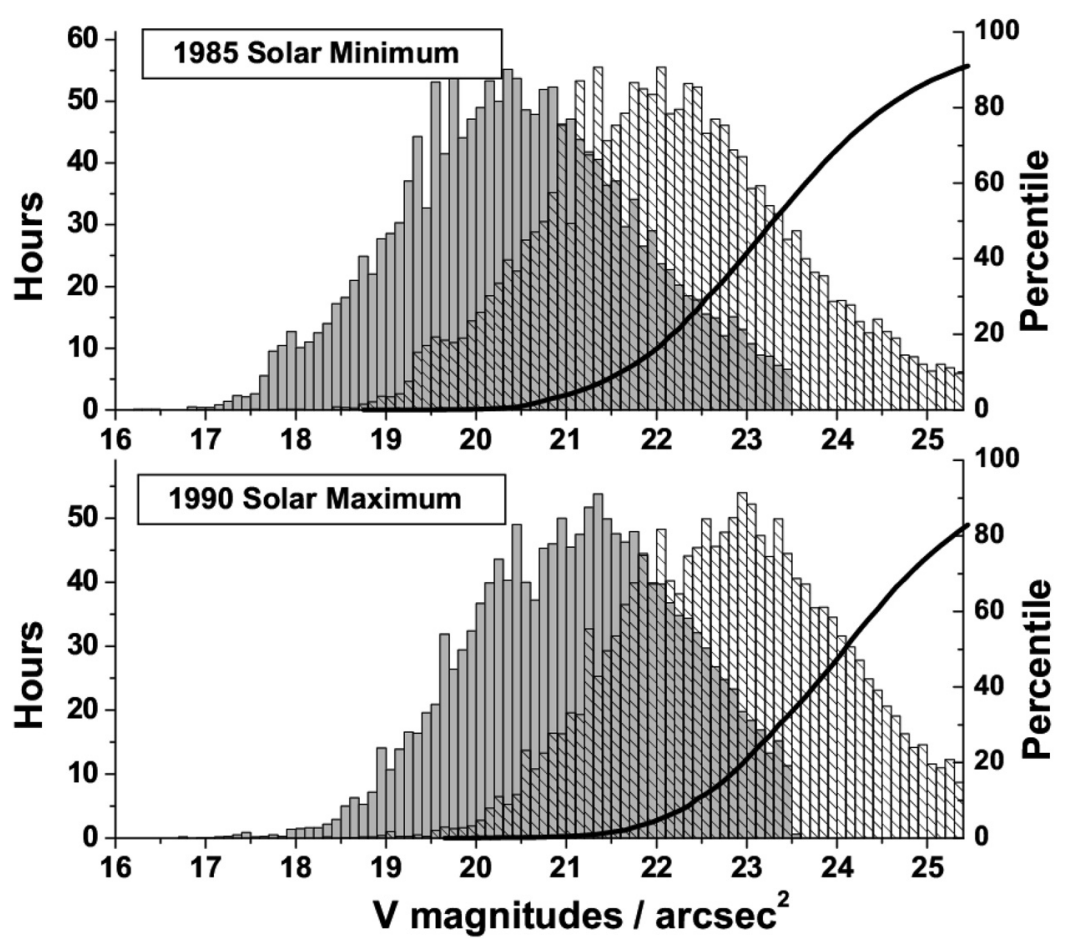

Figure 9 Sky brightness observed at the South Pole (in $V$ magnitudes per square arcsecond) for a standard $V$ filter (solid grey line) and for a notch filter that reduces the photon flux from the 557.7-nm oxygen line to negligible levels (hatched line). The cumulative probability for the filter histogram is shown on the right-hand axis.

1985) of the average electron flux into the upper atmosphere above all three sites. Models by Vallance Jones (1974) permit conversion of electron flux to an estimate of the auroral intensity that would be observed at the ground. This estimate can then be used to calculate the contribution that aurorae would make to the $B$ and $V$ sky background intensity at a given site.

Hardy et al. (1985) presented a statistical study of the Defense Meteorological Satellite Auroral Program over Antarctica. This study aimed to determine the average characteristics of auroral electron precipitation as a function of magnetic local time (MLT), magnetic latitude, and geomagnetic activity as indicated by the geomagnetic $K \mathrm{p}$ index. The $K \mathrm{p}$ index is an index used to describe the activity level of the global geomagnetic field, expressed on a quasi-logarithmic scale between 0 (low magnetic disturbance) and 9 (high magnetic disturbance). Although high figures of geomagnetic $K \mathrm{p}$ cannot be directly related to auroral brightness, during geomagnetically disturbed times aurorae are generally more frequent. Assuming an average level of auroral activity $(K \mathrm{p}=4)$ and with a knowledge of the geomagnetic positions of the South Pole, Dome $\mathrm{C}$, and Dome A, an estimate can be made of the typical electron flux into these sites as a function of MLT. These values are shown in Table 9.

Several theoretical models have been derived to relate the incident electron flux to the intensity of the aurora that it produces. Using the model described by Vallance Jones (1974), we can use the average electron fluxes from Hardy et al. (1985) to arrive at an estimate of the auroral
Table 9. Average electron flux in $10^{7} \mathrm{keV} \mathrm{cm}^{-2} \mathrm{~s}^{-1} \mathrm{sr}$

\begin{tabular}{lcrcc}
\hline MLT $^{1}$ & $6 \mathrm{~h}$ & $12 \mathrm{~h}$ & $18 \mathrm{~h}$ & $24 \mathrm{~h}$ \\
\hline SP & 12.14 & 27.14 & 8.93 & 6.07 \\
Dome C & 2.0 & 1.64 & 1.64 & 1.64 \\
Dome A & 6.07 & 10.71 & 2.93 & 1.71
\end{tabular}

${ }^{1}$ The difference between MLT and UT is $3 \mathrm{~h} 43 \mathrm{~min}$ for South Pole (SP), $2 \mathrm{~h} 34 \mathrm{~min}$ for Dome C, and $2 \mathrm{~h} 5 \mathrm{~min}$ for Dome A.

intensity in a particular emission line or band. Vallance Jones (1974) calculates that the electron flux required to produce an IBC3 aurora $\left(I_{557.7}=100 \mathrm{kR}, I_{427.8}=30 \mathrm{kR}\right)$ is $154 \mathrm{erg} \mathrm{cm}^{-2} \mathrm{~s}^{-1} \mathrm{sr}^{-1}$.

The fluxes in Table 9 are given in $\mathrm{keV} \mathrm{cm}^{-2} \mathrm{~s}^{-1} \mathrm{sr}^{-1}$ which were converted to $\mathrm{erg} \mathrm{cm}^{-2} \mathrm{~s}^{-1} \mathrm{sr}^{-1}$ using the conversion factor $1 \mathrm{keV}=1.6 \times 10^{-9} \mathrm{erg}$. The calculations in Section 4.1 estimate that for an IBC3 aurora (which corresponds to an incident electron flux of $154 \mathrm{erg} \mathrm{cm}^{-2} \mathrm{~s}^{-1} \mathrm{sr}^{-1}$ ), the resulting auroral intensity in $B$ band is equal to $17.6 \mathrm{mag} \operatorname{arcsec}^{-2}$. If we know the electron flux, $F_{\mathrm{E}}$, and we wish to ascertain $I_{B}$, the corresponding auroral emission intensity in the $B$ band, we can calculate it using the following relation:

$$
I_{B}=-2.5 \log _{10}\left(\frac{F_{\mathrm{E}}}{154}\right)+17.6 .
$$

At South Pole, an incident electron flux of $12.14 \times 10^{7} \mathrm{keV}$ $\mathrm{cm}^{-2} \mathrm{~s}^{-1} \mathrm{sr}^{-1}$ (from Table 9) will result in a $B$ band auroral 
Table 10. Sky background in units of $B$ and $V$ magnitudes $\operatorname{arcsec}^{-2}$ produced by aurora at the South Pole, derived from the incident electron flux levels shown in Table 9

\begin{tabular}{lllll}
\hline MLT & $6 \mathrm{~h}$ & $12 \mathrm{~h}$ & $18 \mathrm{~h}$ & $24 \mathrm{~h}$ \\
\hline$B$ & 24.9 & 24.1 & 25.3 & 25.7 \\
$V$ & 23.6 & 22.7 & 23.9 & 24.3 \\
\hline
\end{tabular}

Table 11. The difference (in magnitudes) between the auroral brightness of the sky at Dome $C$ and Dome $A$, relative to that at the South Pole; values are calculated as a function of MLT, for an average level of auroral activity

\begin{tabular}{lcccc}
\hline MLT & $6 \mathrm{~h}$ & $12 \mathrm{~h}$ & $18 \mathrm{~h}$ & $24 \mathrm{~h}$ \\
\hline Dome C & 2.0 & 3.1 & 1.8 & 1.4 \\
Dome A & 0.8 & 1.0 & 1.2 & 1.4 \\
\hline
\end{tabular}

emission intensity of $24.9 \mathrm{mag} \operatorname{arcsec}^{-2}$. The calculations in Section 4.2 estimate that for an IBC3 aurora in the $V$ band, an incident electron flux of $154 \mathrm{erg} \mathrm{cm}^{-2} \mathrm{~s}^{-1} \mathrm{sr}^{-1}$ produces an auroral emission intensity in $V$ band of $16.4 \mathrm{mag} \operatorname{arcsec}^{-2}$. Therefore, for the $V$ band, we can calculate the emission intensity, $I_{V}$, using the following equation:

$$
I_{V}=-2.5 \log _{10}\left(\frac{F_{\mathrm{E}}}{154}\right)+16.4
$$

Thus, an electron flux of $12.14 \times 10^{7} \mathrm{keV} \mathrm{cm}^{-2} \mathrm{~s}^{-1} \mathrm{sr}^{-1}$ will produce an emission intensity in $V$ band of $23.6 \mathrm{mag} \operatorname{arcsec}^{-2}$. Using Equation (3) and the average electron fluxes from Hardy et al. (1985), we can compare the total contribution that auroral emission makes to the $B$ and $V$ bands at the three sites. Table 10 shows the sky background brightness at South Pole, in units of $B$ and $V$ magnitudes per square arcsecond. Table 11 shows the relative auroral background magnitude at Dome $\mathrm{C}$ and Dome A compared with that at the South Pole. In regions of high auroral activity, such as the South Pole, emission intensities are also dependent on MLT (Vallance Jones 1974). Therefore these comparisons are shown for four times during a $24 \mathrm{~h}$ period.

The rate of auroral emission varies as a function of height, and an intensity observed at the ground is integrated along a line of sight. Because auroral structures are aligned along magnetic field lines, observations viewed away from the magnetic zenith will intersect auroral emissions of different intensities, thicknesses, and height distributions (Omholt 1971; Vallance Jones 1974). All line and band emission intensities discussed here are for those viewed towards the magnetic zenith.

South Pole, located within the auroral zone, experiences auroral activity with a greater frequency than does Dome A and Dome C. The close proximity of Dome C to the geomagnetic pole results in a sky background that is darker by up to $3.1 \mathrm{mag}$. Dome A experiences less auroral intensity than the South Pole, but more than Dome C.

\section{Discussion}

Dome C (Candidi \& Lori 2003) has excellent prospects as a site for astronomical observations in a variety of wavelength regions from the submillimetre to the optical. The advantages of Dome $\mathrm{C}$ include its low water-vapour content, infrared sky background emission 20-100 times lower than temperate sites, and exceptional atmospheric stability (Storey et al. 2003). Daytime (summer) measurements show that ground-level seeing is good (Aristidi et al. 2003), while wind speeds and atmospheric turbulence during winter are extremely low (Travouillon et al. 2003), indicating that wintertime seeing is likely to be exceptional. This has recently been confirmed by Lawrence et al. (2004). These advantages make Dome $\mathrm{C}$ a candidate for an extremely large telescope (ELT), as well as a potential site for optical and infrared interferometry.

To evaluate the effect on optical astronomy at Dome C, we now consider the contribution auroral emissions could make to Dome $\mathrm{C}$ sky brightness. Figures 10 and 11 plot the wintertime auroral intensities in the $B$ and $V$ bands at Dome $C$, by rescaling the South Pole $B$ and $V$ band intensities in Section 4 with the relative auroral intensities in Section 5. In $B$ band, for $50 \%$ of the wintertime in a solar minimum year, Figure 10 indicates that the auroral sky background is less than $24.0 \mathrm{~B} \mathrm{mag} \operatorname{~arcsec}^{-2}$, whereas during a solar maximum year, the auroral sky background is below $22.9 \mathrm{~B} \mathrm{mag} \mathrm{arcsec}{ }^{-2}$ for $50 \%$ of the winter. In $V$ band, Figure 11 indicates that the auroral sky background contribution during a solar maximum year is less than $22.7 \mathrm{mag} \mathrm{arcsec}^{-2}$ for $50 \%$ of the winter time, whereas during a solar minimum, the background in $V$ is $23.5 \mathrm{mag} \operatorname{arcsec}^{-2}$. The use of notch filters, as discussed in Section 5, could reduce the auroral sky background contribution further still.

We now examine the science case for one of the proposed ELTs, the Giant Magellan Telescope (GMT), and ask what impact the aurorae would have on key projects. A second GMT has already been proposed for Dome C (Angel, Lawrence, \& Storey 2004). The GMT design consists of a segmented primary mirror with an effective collecting area of $21.5 \mathrm{~m}$, with a $3.5-\mathrm{m}$ Gregorian secondary mirror. The various operating modes for the GMT would have limiting resolutions ranging from natural seeing, down to 0.007 arcsec for a full multi-conjugate adaptive optics (AO) system that uses natural and laser guide stars. Further details of the telescope, along with science priorities, can be seen at the GMT homepage (www. gmto.org).

Most of the science goals of the GMT focus on nearIR and mid-IR wavebands, as these are wavelengths where high-order adaptive optics $(\mathrm{AO})$ permit diffraction-limited observations. Beyond $800 \mathrm{~nm}$ (where $\mathrm{OH}$ airglow lines begin to dominate the spectrum at mid-latitude sites), the sky background emission at Dome $\mathrm{C}$ is not yet characterised, but the same methods that are used to remove 


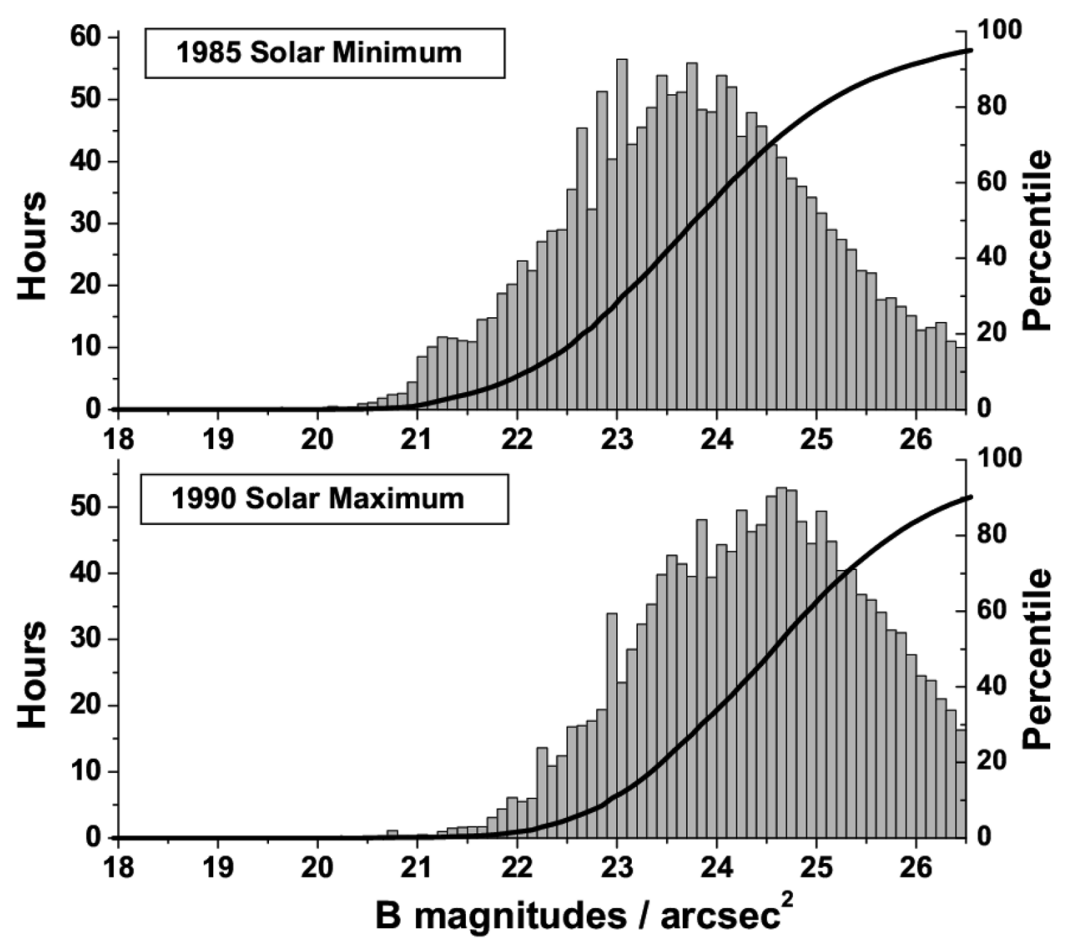

Figure 10 Calculated auroral contribution to sky brightness at Dome $\mathrm{C}$ (in $B$ magnitudes per square arcsecond). The cumulative probability is referenced to the right-hand axis.

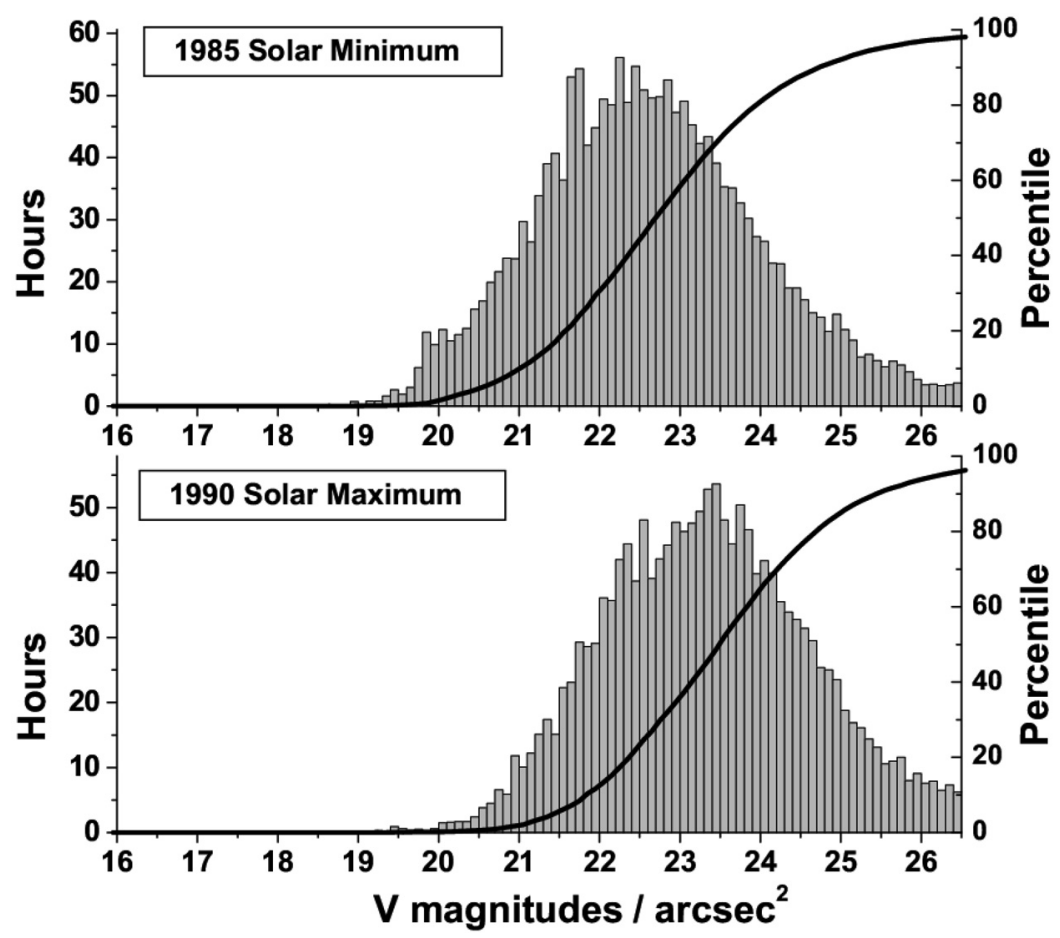

Figure 11 Calculated auroral contribution to sky brightness at Dome $\mathrm{C}$ (in $V$ magnitudes per square arcsecond). The cumulative probability is referenced to the right-hand axis.

aurorae from observations could also be used to remove airglow.

A majority of the science projects that are guiding the design of the GMT place emphasis on highresolution spectroscopy. Narrow auroral emission lines can be removed from spectra, while the reduced slit width of the instrument made possible by the better seeing conditions at Dome $\mathrm{C}$ will further decrease the sky background.

a) Intergalactic medium: Distant quasar observations using high-resolution spectroscopy permit comparisons 
of the intergalactic medium along different lines of sight. Most quasars are reasonably bright, at 15 to $17 \mathrm{~V}$ magnitudes, and high-resolution spectroscopy should permit the easy identification and removal of the narrow auroral lines in the spectra.

b) Multi-aperture spectrographs: Redshift galaxy surveys are looking to collect continuum spectra of galaxies down to $26 I$ magnitudes, in $15^{\circ}$ fields at intermediate resolutions. Slit spectroscopy would allow better sky subtraction than fibre-fed spectrographs, but auroral light in the continuum spectra could prove a limiting factor.

c) Integral field spectroscopy: Integral field spectroscopy of nearby galaxies can determine stellar populations, orbital structure, and the kinematics around nuclear black holes. Spectroscopic mapping allows discrete auroral lines in the galactic spectra to be removed prior to integrating the field and is already carried out with bright sky lines. These observations should not be affected by aurorae at Dome C.

d) Faint-end AGN: Broad emission lines in AGN are black hole signatures which, even at modest redshifts, are lost in the light of their host galaxies. Using an ELT in a high resolution mode and masking strong telluric $\mathrm{OH}$ emission features, the spectra can be rebinned at lower resolution to improve the sensitivity of these weak broad lines. Removing the light from the host galaxy will be the primary limiting factor in these observations, not aurorae.

e) Resolved stellar populations: Imaging in the near-IR with large aperture telescopes will extend the study of stellar environments that are currently confusion limited. These observations will be limited to the seeing that can be achieved at an observing site and on the AO capabilities of the telescope. Auroral sky background should not be a factor with such measurements.

f) Single object, deep field studies: Continuum measurements and imaging of faint objects may be affected by a fast-varying background and a large number of contributing sky lines within the band. Such projects include measuring abundances of the supergiants and the studies of the abundance of red giants in the Local Group. Long exposures would be affected by short timescale variations in the background.

g) Extrasolar planets: Circumstellar debris disks could be probed down to scales less than $10 \mathrm{AU}$ with a GMT. The better seeing at Dome $C$ reduces the sky background per pixel (as the pixel size can be scaled with the resolution) and means that the flux from the sky will be lower in seeing-limited observations.

h) Direct detection of young planets: An ELT would allow direct detection of planets close to a parent star (using coronography and apodisation, or nulling interferometry). Both of these methods require AO to reach the resolution required (60-120 milliarcsec in $J$ band) at a temperate site, whilst at Dome $\mathrm{C}$ such resolutions could be achieved in natural seeing conditions. The light from the parent star is the fundamental limit to such observations, and so additional sky background from aurorae will make little difference to these measurements.

\section{Conclusions}

At sites such as Skibotn and South Pole Station, intense auroral emissions produce an increase in sky background brightness over all three visible wavebands $(V, B$, and $R)$. For photometric observations, the variability of the background during short time periods (Myrabo 1980) requires careful and frequent sky background measurement and subtraction. With spectroscopy, the positions of these emission lines may prevent observations of specific lines within observed sources, but otherwise should present little problem.

For an aurora in the $B$ band, a notch filter can eliminate the $427.8-\mathrm{nm} \mathrm{N}_{2}^{+}$vibrational band emission and reduce the auroral contribution to the sky background by $0.7 \mathrm{~B}$ mag. Within the $V$ band, a notch filter at $557.7 \mathrm{~nm}$ can decrease the auroral contribution to the sky background by $1.6 \mathrm{~V}$ mag. Specially designed filter passbands could give significantly darker sky backgrounds than with standard $B$ and $V$ filters. However, a fully optimised Rugate filter could yield greater improvements yet. Projects such as the Vulcan South Transit Search have avoided using wavelengths of strong and variable auroral emission lines by using carefully selected filter passbands.

A site such as Dome C, located higher on the Antarctic Plateau and closer to the geomagnetic pole, will be more favourable for optical astronomy as the auroral contribution to sky brightness is reduced by up to $3.1 \mathrm{mag}$ when compared with that at the South Pole.

\section{Acknowledgments}

We gratefully acknowledge the generosity of D. L. Detrick and T. J. Rosenberg at the University of Maryland, and A. T. Weatherwax at Siena College, in providing the South Pole photometer data used in this study. That research was partially supported by the National Science Foundation under grant OPP-0003881 to the University of Maryland. We thank S. Mende for his advice. We extend our gratitude to D. Caldwell for information used in this paper, and to D. Hrubes for his advice from South Pole. We would also like to thank M. C. B. Ashley, M. G. Burton, J. K. Webb, and J. S. Lawrence for helpful discussions. We are indebted to G. Burns of the Australian Antarctic Division for insightful analysis and assistance. This paper has been greatly improved by the suggestions of an anonymous referee. This work was made possible by funding from the Australian Research Council and the Australian Antarctic Division.

\section{References}

Angel, J. R., Lawrence, J., \& Storey, J. W. V. 2004, SPIE, 5382, 76 Aristidi, E., Agabi, A., Vernin, J., Azouit, M., Martin, F., Ziad, A., \& Fossat, E. 2003, A\&A, 406, 19

Baker, D. J., \& Romick, G. J. 1976, ApOpt, 15, 1966 
Bashkin, S., Thiede, D. A., Sercel, P. C., Lin, P. C., \& Traebert, E. 1991, PhRvA, 43, 3553

Benn, C. R., \& Ellison, S. L. 1998, NewAR, 42, 503

Bessel, M. S. 1979, PASP, 91, 589

Boccas, M., Ashley, M. C. B., Phillips, A., Schinckel, A., \& Storey, J. W. V. 1998, PASP, 110, 306

Candidi, M., \& Lori, A. 2003, MmSAI, 74, 29

Caldwell, D. A., Borucki, W. J., Showen, R., Jenkins, J., Doyle, L., Ninkov, Z., \& Ashley, M. C. B. 2002, in IAU Symp. S-213, Bioastronomy 2002: Life among the Stars, eds. R. P. Norris, \& F. H. Stootman (San Francisco: ASP), p. 93

Carroll, J. J., \& Fitch, B. W. 1981, JGR, 86, 5271

Chamberlain, J. W. 1961, Physics of the Aurora and Airglow, International Geophysics Series (New York: Academic Press)

Gattinger, R. L., \& Vallance Jones, A. 1974, CaJPh, 52, 2343

Gattinger, R. L., Vallance Jones, A., Hecht, J. H., Strickland, D. J., \& Kelly, J. 1991, JGR, 96, 11341

Hardy, D. A., Gussenhoven, M. S., \& Holeman, E. 1985, JGR, 90,4229

Johnson, W. E., \& Crane, R. L. 1993, SPIE, 2046, 88
Lawrence, J. S., Ashley, M. C. B., Tokovinin, A., \& Travouillon, T. 2004, Natur, 431, 278

MacMillan, S., \& Quinn, J. M. 2000, EP\&S, 52, 1149

McHarg, M. G. 1998, GeoRL, 25, 2637

Myrabo, H. K. 1979, Ap\&SS, 60, 367

Myrabo, H. K. 1980, A\&A, 84, 297

Omholt, A. 1971, The Optical Aurora (Berlin: Springer)

Paxton, L. J., \& Meng, C.-I. 1999, Johns Hopkins Technical Digest, 20, 556

Sharp, W. E. 1978, JGR, 83, 4373

Storey, J. W. V., Ashley, M. C. B., \& Burton, M. G. 1996, PASA, 13,35

Storey, J. W. V., Ashley, M. C. B., Lawrence, J. S., \& Burton, M. G. 2003, MSAIS, 2, 13

Swenson, G. R., Rairden, R. L., Solomon, S. C., \& Anath, S. 1998, ApOpt, 37, 5760

Travouillon, T., Burton, M. G., Ashley, M. C. B., Lawrence, J., \& Storey, J. W. V. 2003, MSAIS, 2, 150

Trondsen, T. S. 1998, PhD Thesis, University of Tromso, Norway Vallance Jones, A. 1974, Aurora (Dordrecht: Reidel) 